\title{
IDENTIFICATION OF FASCIOLIDS AT DIFFERENT ALTITUDES OF AZAD JAMMU AND KASHMIR
}

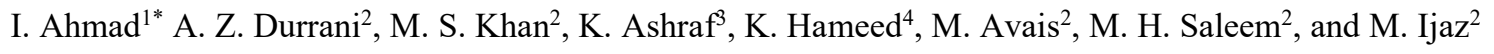 \\ ${ }^{1}$ Department of Veterinary Clinical Sciences, University of the Poonch, Rawalakot, Azad Kashmir, ${ }^{2}$ Department of \\ Clinical Medicine and Surgery, ${ }^{3}$ Department of Parasitology, University of Veterinary and Animal Sciences, Lahore, \\ Pakistan. ${ }^{4}$ Mirpur University of Science and Technology, Mirpur Azad Kashmir, Pakistan. \\ *Corresponding Author's email: vetmedicine2015@gmail.com
}

\begin{abstract}
Fasciolosis is an important zoonotic disease affecting domestic animals in most parts of the world. Two important species of flukes Fasciola (F.) gigantic and Fasciola hepatica with an intermediate form was reported by many researchers from different countries of the world. For identification of species of fasciolids 167 flukes were collected from domestic animals at abattoirs from three topographic locations from three districts of Azad Jammu and Kashmir during 2012. Readings were taken using glass rulers and ocular micrometer. Characterization of $F$. hepatica revealed average length $(\mathrm{mm}) 20.66 \pm 2.27$ to $23.20 \pm 5.25$ and width $9.90 \pm 0.50$ to $10.16 \pm 1.00$ and that of $F$. gigantic $41.25 \pm 1.55$ to $45.33 \pm 0.83,9.44 \pm 0.20$ to $9.71 \pm 0.37$ respectively. It was noted that $F$. hepatica predominantly affecting animals at high altitudes with cooler climate and F. gigantica at low altitudes with warm conditions. No "intermediate form" of fasciola was found at any location or host animal. All flukes after morphometery were identified through PCR using species specific primers. Further investigations are required to investigate intermediate form from different geographic locations of Pakistan for control measures.
\end{abstract}

Keywords: Fasciola species; Morphometery; Altitudes; topographic locations; Azad Kashmir https://doi.org/10.36899/JAPS.2020.3.0090 Published online March 25, 2020

\section{INTRODUCTION}

Fasciolosis is an important human health concern affecting domestic and wild animals in many parts of the world (Iyiola et al. 2018). F. hepatica was reported from temperate and $\mathrm{F}$. gigantica from tropical zones, overlapped in subtropical areas (Abdulwahed and Al-Amery 2019). Human infection occurs accidentally most frequently in sheep and cattle rearing families. Proper identification based on morphology of species is necessary for understanding pathogenesis. Moreover use of molecular techniques was helpful to identify intermediate subspecies (Shahbakhsh et al. 2016). Both species have different epidemiological characteristics where $F$. hepatica is less pathogenic than $F$. gigantic. The reason is smaller in size instead of genetic characters. Pathogenesis may be misleading where intermediate form is present and may have significant complications in the area (Aryaeipour et al. 2017). Identification of species can be made on the basis of morphology or molecular analysis however, microscopic analysis is recommended. Molecular techniques have greater sensitivity but not cost effective for routine use (El-Rahimy et al. 2012). Characterization of fasciola species is useful for epidemiology, prevention and control of disease in endemic areas where hybridization phenomenon occurs (Akhlaghi et al. 2017). Such hybrids/ intermediate forms were reported from Korea
(Agatsuma et al. 2000), Iran (Amor et al. 2011), Veitnam (Le et al. 2008), Egypt (Amer et al. 2011) and China (Peng et al. 2009). Recent studies found F. gigantica to be more pathogenic and widespread in Pakistan (Afshan, et al 2014). Accurate diagnosis of the disease needs identification of the species of the fluke involved for assessment of the risk factors and control strategy (Khan et al. 2009). Data on morphometric and molecular identification at species level are lacking in Pakistan which prompted this study to be carried out for morphometric measurements of the two species and conformation through PCR.

\section{MATERIALS AND METHODS}

Geographic locations and sampling: Adult liver fluke samples $(n=167)$ were collected from affected livers of sheep, buffalo, cattle and goats. These sample were collected from three districts (Table 1\&2) based on altitudes, A) Mirpur (<3000 feet), B) Neelam (>6000) and C) Poonch (3000-6000). Collected samples were preserved in physiological saline immediately after removal from bile duct, labelled and shifted to University of Health Sciences, Lahore for phenotypic and genetic identification.

Morphometric measurements: Flukes were flattened and clamped between two glass slides, fixed with formalin $(10 \%)$ and kept in refrigerator. After 12 
hours specimen were washed to remove debris and traces of formalin and separated from slides. Borex caramine staining was done and dehydrated for 15 minutes each in 70\%, 80\%, 95\% and absolute alcohol. Samples were mounted in Canadabalsam as described by Periago et al. (2006). Measurements of specimen included body length, width, diameter of suckers and distance between oral and ventral suckers were made using eye piece micrometer and glass ruler. The average of each parameter was calculated along with standard error.

Molecular identification: Specimens collected from three topographic locations $(n=50)$ were used for PCR. Specimens already identified on the basis of morphometery were fixed in ethanol until DNA extraction. GeneAll DNA extraction kit (GeneAll Biotechnology Co. Ltd, Korea) was used for extraction. PCR was done with specific primers reported by (McGarry et al. 2007).

PCR Conditions: Denaturation $94^{\circ} \mathrm{C}$ for $1 \mathrm{~min}$. Annealing $52^{\circ} \mathrm{C}$ for $1 \mathrm{~min}$. Extension $72^{\circ} \mathrm{C}$ for $2 \mathrm{~min}$. followed by $10 \mathrm{~min}$ of final extension at $72^{\circ} \mathrm{C}$.

\section{RESULTS}

Mean worm load per liver in small and large ruminants was 11.92 with maximum load of 22 . The average worm count of $F$. hepatica depicted 10.37 as compared to 14 for $F$. gigantic.
Both species were found overlapped at lower altitudes and warm climate. $F$. gigantic was not encoutered at high altitude however, $F$. hepatica found present in livers of infected animal at all topographic locations. $F$. hepatica measurements revealed body length $20.55 \pm 1.09$ to $22.73 \pm 2.01 \mathrm{~mm}$ at altitude $>6000$ feet, $19.81 \pm 1.35$ to $22.50 \pm 2.73 \mathrm{~mm}$ at altitude 3000 6000 feet and $20.66 \pm 2.27$ to $23.2 \pm 5.25 \mathrm{~mm}$ at altitude $<3000$ feet (Table 1). Body length of $F$. gigantic was $42.23 \pm 0.95$ to $45.33 \pm 0.83 \mathrm{~mm}$ at altitude $3000-6000$ feet and $41.25 \pm 1.55$ to $41.54 \pm 1.68 \mathrm{~mm}$ at $<3000$ feet (Table 2 ). The body length showed a marked difference in the size of the two species. The body width of $F$. hepatica ranged from $9.90 \pm 0.50 \mathrm{~mm}$ to $10.16 \pm 1.00 \mathrm{~mm}$ as compared to $9.44 \pm 0.20$ to $9.71 \pm 0.37 \mathrm{~mm}$ of $F$. gigantica. The diameters of suckers and distance between them were not found to be a reliable tool for differentiation however, distance between sucker (ventral) and taper end of the body was quite different in two species. This value for $F$. hepatica was $19.83 \pm 5 \mathrm{~mm}$ as compared to F.gigantica37.65 $\pm 1.4 \mathrm{~mm}$.

PCR product revealed 391 bp sizes for $F$. hepatica with specific primer and did not generate any product when DNA of $F$. gigantica was used. DNA of $F$. gigantic generated $235 \mathrm{bp}$ amplification product with specific primer and no product generated using non specific primer (Fig. 1). Hence Fasciola species identified on the basis of morphological parameters were confirmed with PCR.

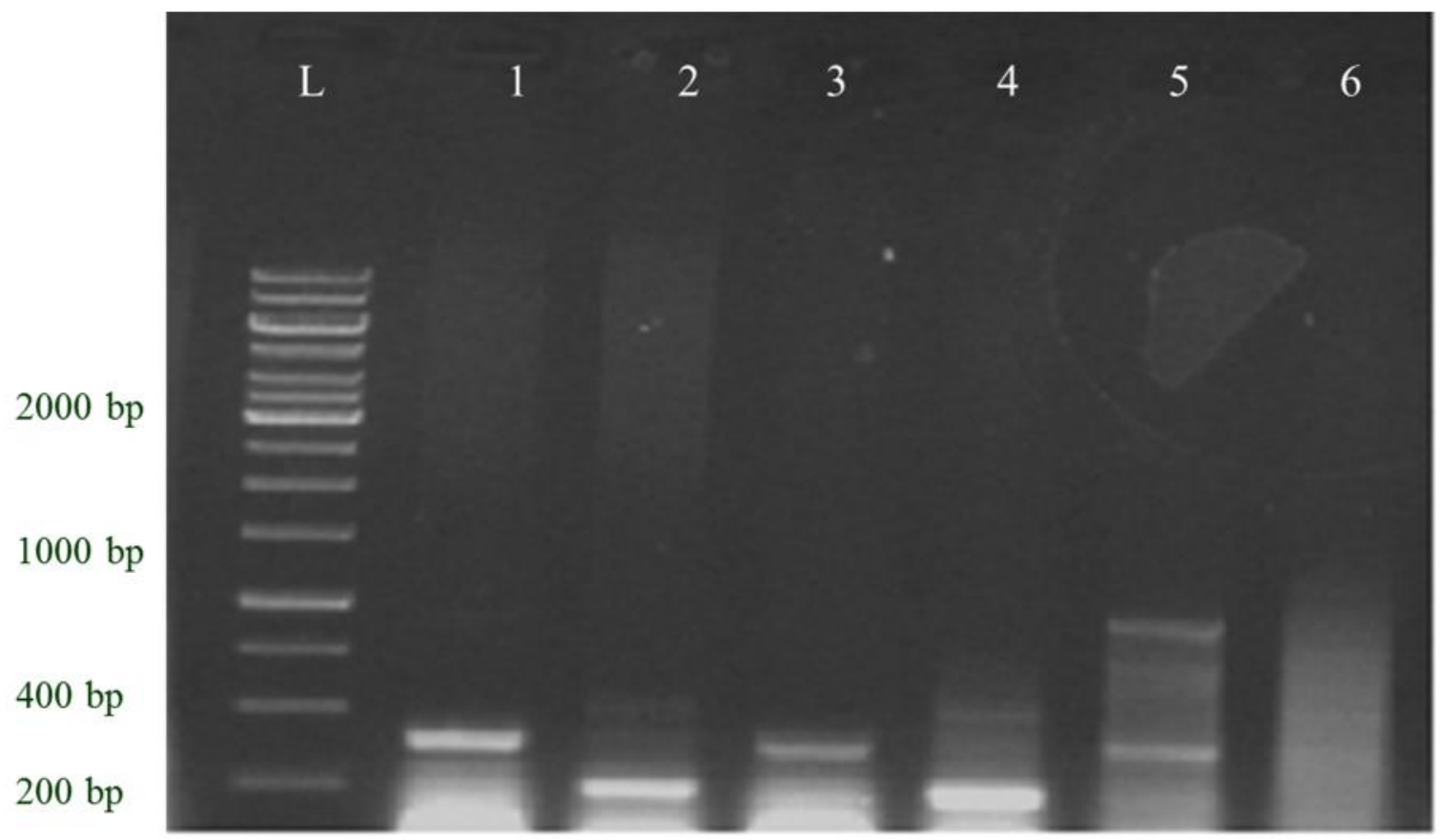

Figure 1. Amplification of DNA of fasciola species in AJK.

Lane 1. F.hepatica sheep Lane 2. F.gigantica goat Lane 3. F.hepatica goat Lane 4.F.gigantica Buffalo Lane 5. F. hepatica sheep Lane 6. Control -ve 
Table 1. Morphometery of Fasciola hepatica at different geographic locations and hosts in AJK

\begin{tabular}{|c|c|c|c|c|c|c|c|c|c|c|}
\hline GA & D & Alt & HS & NF & $\begin{array}{c}\text { BL } \\
\operatorname{Avg} \pm S E\end{array}$ & $\begin{array}{c}\text { BW } \\
\operatorname{Avg} \pm \text { SE }\end{array}$ & $\begin{array}{c}\text { OS } \\
\operatorname{Avg} \pm \text { SE }\end{array}$ & $\begin{array}{c}\text { VS } \\
\operatorname{Avg} \pm \text { SE }\end{array}$ & $\begin{array}{c}\text { D-OS/VS } \\
\operatorname{Avg} \pm \text { SE }\end{array}$ & $\begin{array}{c}\text { D-VS/P } \\
\text { Avg } \pm \text { SE }\end{array}$ \\
\hline Sharda & Neelam & 6499 & Goat & 22 & $21.32 \pm 1.14$ & $10.05 \pm 0.24$ & $0.76 \pm 0.008$ & $1.27 \pm 0.02$ & $1.40 \pm 0.06$ & $17.93 \pm 1.16$ \\
\hline Machal & Neelam & 7350 & Sheep & 08 & $22.73 \pm 2.01$ & $10.13 \pm 0.48$ & $0.83 \pm 0.01$ & $1.31 \pm 0.04$ & $1.21 \pm 0.17$ & $19.63 \pm 2.14$ \\
\hline Ghamoot & Neelam & 7800 & Cow & 17 & $20.55 \pm 1.09$ & $10.15 \pm 0.11$ & $0.73 \pm 0.01$ & $1.29 \pm 0.02$ & $1.43 \pm 0.09$ & $17.53 \pm 1.25$ \\
\hline Rawalakot & Poonch & 5374 & Sheep & 14 & $19.81 \pm 1.35$ & $10.05 \pm 0.20$ & $0.71 \pm 0.01$ & $1.25 \pm 0.03$ & $1.24 \pm 0.06$ & $16.83 \pm 1.27$ \\
\hline Rawalakot & Poonch & 5374 & Cow & 08 & $21.51 \pm 1.88$ & $10.06 \pm 0.30$ & $0.75 \pm 0.04$ & $1.33 \pm 0.04$ & $1.39 \pm 0.14$ & $18.32 \pm 2.17$ \\
\hline Hajeera & Poonch & 3168 & Goat & 05 & $22.50 \pm 2.73$ & $9.90 \pm 0.50$ & $0.69 \pm 0.06$ & $1.20 \pm 0.07$ & $1.31 \pm 0.21$ & $19.30 \pm 2.73$ \\
\hline Mirpur & Mirpur & 1503 & Goat & 03 & $23.20 \pm 5.25$ & $10.16 \pm 1.00$ & $0.74 \pm 0.08$ & $1.31 \pm 0.11$ & $1.47 \pm 0.43$ & $19.83 \pm 5.21$ \\
\hline Mirpur & Mirpur & 1503 & Calf & 06 & $20.66 \pm 2.27$ & $10.0 \pm 0.51$ & $0.72 \pm 0.03$ & $1.34 \pm 0.04$ & $1.38 \pm 0.20$ & $17.60 \pm 2.79$ \\
\hline
\end{tabular}

GA: Geographical Area; D: District; Alt: Altitude (feet); HS: Host Species; NF: Number of Flukes; BL: Body Length; BW: Body Width; OS: Oral Sucker; VS: Ventral Sucker;3

D-OS/VS: Distance between OS/VS, D-VS/P Distance between VS \& posterior end of the body; (Measurements in mm)

Table 2. Morphometery of Fasciola gigantica at different geographic locations and hosts in AJK

\begin{tabular}{|c|c|c|c|c|c|c|c|c|c|c|}
\hline GA & D & Alt & HS & NF & BL & BW & OS & VS & D-OS/VS & D-VS/P \\
\hline & & & & & $\operatorname{Avg} \pm \mathrm{SE}$ & $\mathrm{Avg} \pm \mathrm{SE}$ & $\mathrm{Avg} \pm \mathrm{SE}$ & $\operatorname{Avg} \pm \mathrm{SE}$ & $\mathrm{Avg} \pm \mathrm{SE}$ & $\operatorname{Avg} \pm \mathrm{SE}$ \\
\hline Mirpur & Mirpur & 1503 & Cow & 13 & $41.54 \pm 1.68$ & $9.64 \pm 0.38$ & $0.94 \pm 0.04$ & $1.60 \pm 0.07$ & $1.67 \pm 0.06$ & $38.21 \pm 1.62$ \\
\hline Dadyal & Mirpur & 1503 & Goat & 12 & $41.50 \pm 1.12$ & $9.71 \pm 0.37$ & $0.91 \pm 0.02$ & $1.48 \pm 0.07$ & $1.67 \pm 0.08$ & $38.25 .9 \pm 1.37$ \\
\hline Rawalakot & Poonch & 5374 & Goat & 21 & $42.23 \pm 0.95$ & $9.44 \pm 0.20$ & $0.89 \pm 0.01$ & $1.36 \pm 0.04$ & $1.57 \pm 0,06$ & $38.73 \pm 0.98$ \\
\hline Khaigala & Poonch & 5500 & Cow & 10 & $43.50 \pm 1.32$ & $9.50 \pm 0.12$ & $0.87 \pm 0.04$ & $1.62 \pm 0.09$ & $1.76 \pm 0.07$ & $39.87 \pm 1.37$ \\
\hline Hajeera & Poonch & 3168 & Buffalo & 19 & $45.33 \pm 0.83$ & $9.66 \pm 0.25$ & $0.85 \pm 0.02$ & $1.71 \pm 0.07$ & $1.94 \pm 0.06$ & $41.73 \pm 0.91$ \\
\hline Jatlan & Mirpur & 1377 & Goat & 09 & $41.25 \pm 1.55$ & $9.57 \pm 0.42$ & $0.92 \pm 0.51$ & $1.64 \pm 0.12$ & $1.62 \pm 0.08$ & $37.65 \pm 1.48$ \\
\hline
\end{tabular}

between OS/VS, D-VS/P Distance between VS \& posterior end of the body;(Measurements in mm) 


\section{DISCUSSION}

Livestock farming is important in sustainable economic development in developing world. Fasciolosis is a serious challenge to livestock particularly sheep and goats farming worldwide because of its high occurrence. Moreover incidence of human infection is high in some countries of high animal infections (Amer et al. 2016). Being a zoonotic disease with limitations in drugs availability for treatment control measures are urgently needed (Knubben-Schweizer and Torgerson 2015). Data on genetic and phenotypic characteristics of Fasciola species is limited in the state of AJK. Present study elucidated such characteristics of Fasciola species infecting large and small ruminants from different localities. Morphometric features of two species were studied to identify presence of hybrid form where overlapping occurs. Parameters like specimen length, width, diameter of two suckers were considered practical criteria for differentiation between species of liver flukes (Akhlaghi et al. 2017). Results of present study indicated that measurements differed considerably between two species at three different topographic locations. Comparable results were reported from different host animals (Ashrafi et al. 2006) and (Yakhchali et al. 2015).

Microscopic measurements are helpful in characterization of liver flukes in areas with low occurrence. However, in countries where presence of intermediate forms such as Japan and Korea cannot be classified as $F$. gigantica and $F$. hepatica (Itagaki et al. 2005). Hybrids of two species were listed by different researchers (Afshan et al 2014; Ai et al. 2010; Itagaki et al. 2009; Ali et al. 2008).

In Asian countries like Pakistan overlap occurs in areas of different altitudes with movements of animals in summer and winter. Prompt control strategies are required to asses overlap scenario and to identify hybrid forms of Fasciola (Afshan et al. 2014). Present study depicted existence of overlap in two districts but no intermediate form was found at any geographic location. These findings were in agreement with (Mas-Coma et al. 2009), as they found $F$. gigantica and F. hepatica as only species of liver flukes in areas with overlapping. The present study concludes that flukes collected from high altitudes were found as $F$. hepatica and at lower altitudes $F$. gigantic. F. hepatica population decreased as altitude decreased and F.gigantica was not present in animals of district Neelam (high altitudes). However, mixed infection found in animals of district Mirpur (lower altitudes). No intermediate form of the Fasciola was encountered at any geographical location or host species of animals.

\section{REFERENCES}

Abdulwahed, T.K. and A.M. Al-Amery (2019). Morphological and molecular study of Fasciola spp. in sheep in Alkut city. Int. J. Biosci. 14(1): 121-130.

Afshan, K., M.A. Valero, M. Qayyum, R.V. Peixoto, A. Magraner and S. Mas-Coma (2014).Phenotypes of intermediate forms of F. hepatica and F. gigantica in buffaloes from Central Punjab, Pakistan. J. Helminthol. 88(4): 1-10.

Agatsuma, T., Y. Arakawa, M. Iwagami, Y. Honzako, U. Cahyaningsih and S.Y. Kang (2000). Molecular evidence of natural hybridization between Fasciola hepatica and F. gigantica. Parasitol. Int. 49: 231-238.

Ai, L., S.J. Dong, W.Y. Zhang, H.M. Elsheikha, Y.S. Mahmmod, R.Q. Lin, Z.G. Yuan, Y.L. Shi, W.Y. Huang and Z. Q. Zhu (2010). Specific PCR-based assays for the identification of Fasciola species: their development, evaluation and potential usefulness in prevalence surveys. Ann. Trop. Med. Parasitol. 104(1): 65-72.

Akhlaghi, E., M.A. Mohammadi, N. Ziaali, M.R. Baneshi, S. Nasibi, H. Kamyabi, S. Rostami and M.F. Harandi (2017). Morphometric and molecular study of fasciola isolates from ruminants in Iran. Turkiye Parazitol. Derg. 41: 192-197.

Ali, H., L. Ai, H.Q. Song, S. Ali, R.Q. Lin, B. Seyni, G. Issa and X. Q. Zhu (2008). Genetic characterisation of Fasciola samples from different host species and geographical localities revealed the existence of $F$. hepatica and F. gigantica in Niger. Parasitol. Res. 102: 1021-1024.

Amer, S., A. ElKhatam, S. Zidan, Y. Feng and L. Xiao (2016). Identity of Fasciola spp. in sheep in Egypt. Parasites \& Vectors 9:623

Amer, S., Y. Dar, M. Ichikawa, Y. Fukuda, C. Tada and T. Itagaki (2011). Identification of Fasciola species isolated from Egypt based on sequence analysis of genomic (ITS1 and ITS2) and mitochondrial (NDI and COI) gene markers. Parasitol. Int. 60: 5-12.

Amor, N., A. Halajian, S. Farjallah, P. Merella, K. Said and B.B. Slimane (2011). Molecular characterization of Fasciola spp. from the endemic area of northern Iran based on nuclear ribosomal DNA sequences. Exp. Parasitol. 128: 196-204.

Aryaeipour, M., A. Bozorgomid, B. Kazemi, M. Behnia, H. Azizi and M.B. Rokni (2017). Molecular and morphometrical characterization of fasciola species isolated from domestic ruminants in 
Ardabil Province, Northwestern Iran. Iran J. Public Health 46(3): 318-325.

Ashrafi, K., M.A. Valero, M. Panova, M.V. Periago, J. Massoud and S. Mas-Coma (2006). Phenotypic analysis of adults of Fasciola hepatica, Fasciola gigantica and intermediate forms from the endemic region of Gilan. Iran Parasitol. Int. 55: 249-60.

El-Rahimy, H.H., A.M. Mahgoub, N.S.M. El-Gebaly, W.M. Mousa and A.S. Antably (2012). Molecular, biochemical and morphometric characterization of Fasciola species potentially causing zoonotic disease in Egypt. Parasitol. Res. 111(3): 1103-11.

Itagaki, T., K. Sakaguchi, K. Terasaki, O. Sasaki, S. Yoshihara and T. Van Dung (2009). Occurrence of spermic diploid and aspermic triploid forms of Fasciola in Vietnam and their molecular characterization based on nuclear and mitochondrial DNA. Parasitol. Int. 58: 81-85.

Itagaki, T., M. Kikawa, K. Sakaguchi, J. Shimo, K. Terasaki and T. Shibahara (2005). Genetic characterization of parthenogenetic Fasciola sp. in Japan on the basis of the sequences of ribosomal and mitochondrial DNA. Parasitol. 131:679-85.

Iyiola, O.A., O. Shittu, O.A. Owolodun, D. A. Animasaun and A.O. Udeze (2018). Morphometric phenotypes and molecular identification of fasciola species isolated from cattle in Ilorin, North-Central Nigeria. Sri Lankan J. Biol. 3(2): 9-23.

Khan, M.K., M.S. Sajid, M.N. Khan, Z. Iqbal and M.U. Iqbal (2009). Bovine fasciolosis: prevalence, effects of treatment on productivity and cost benefit analysis in five districts of Punjab, Pakistan. Res. Vet. Sci. 87: 70-75.

Knubben-Schweizer, G. and P.R. Torgerson (2015). Bovine fasciolosis: control strategies based on the location of Galba truncatula habitats on farms. Vet. Parasitol. 208:77-83.
Le, T.H., N. Van De, T. Agatsuma, T.G.T. Nguyen, Q.D. Nguyen and D.P. McManus (2008). Human fascioliasis and the presence of hybrid/introgressed forms of Fasciola hepatica and Fasciola gigantica in Vietnam. Int. J. Parasitol. 38: 725-730.

Mas-Coma, S., M.A. Valero and M.D. Bargues (2009). Fasciola lymnaeids and human Fasciolosis with a global overview on disease transmission, epidemiology, evolutionary genetics, molecular epidemiology and control. Adv. Parasitol. 69: 41-146

Mc-Garry, J.W., P.L. Ortiz, J.E. Hodgkinson, I. Goreis and D.J. Williams (2007). PCR-based differentiation of Fasciola species (Trematoda: Fasciolidae), using primers based on RAPDderived sequences. Ann Trop. Med. Parasitol. 101(5):415-421.

Peng, M., M. Ichinomiya, M. Ohtori, M. Ichikawa, T. Shibahara and T. Itagaki (2009). Molecular characterization of Fasciola hepatica, Fasciola gigantica, and aspermic Fasciola sp. in China based on nuclear and mitochondrial DNA. Parasitol. Res. 105: 809-15.

Periago, M.V., M.A. Valero, M. Panova and S. MasComa (2006). Phenotypic comparison of allopatric populations of Fasciola hepatica and Fasciolagigantica from European and African bovines using a computer image analysis system (CIAS). Parasitol. Res. 99: 368-378.

Shahbakhsh, M., R. Nabavi and M. Ganjali (2016). Molecular characterization of fasciola samples using sequences of second internal transcribed spacer-rDNA in different geographical localities of Sistan and Balouchestan Province, Iran. Int. J. Enteric Pathog. 4(1): e33362.

Yakhchali, M., R. Malekzadeh-Viayeh, A. Imani-Baran and K. Mardani (2015). Morphological and molecular discrimination of Fasciola species isolated from domestic ruminants of Urmia city, Iran. Iran J. Parasitol. 10: 46-55. 